\title{
时空大数据背景下并行数据处理分析挖掘的 进展及趋势
}

\author{
关雪峰, 曾宇媚* \\ (武汉大学测绘谣感信息工程国家重点实验室,武汉 430079)
}

\begin{abstract}
摘 要: 随着互联网、物联网和云计算的高速发展, 与时间、空间相关的数据呈现出 “爆炸式” 增长的趋势, 时空大数 据时代已经来临。时空大数据除具备大数据典型的“ $4 \mathrm{~V}$ ”特性外, 还具备丰富的语义特征和时空动态关联特性, 已 经成为地理学者分析自然地理环境、感知人类社会活动规律的重要资源。然而在具体研究应用中, 传统数据处理 和分析方法已无法满足时空大数据高效存取、实时处理、智能挖掘的性能需求。因此,时空大数据与高性能计算/ 云计算融合是必然的发展趋势。在此背景下, 本文首先从大数据的起源出发, 回顾了大数据概念的发展历程, 以及 时空大数据的特有特征; 然后分析了时空大数据研究应用产生的性能需求, 总结了底层平台软硬件的发展现状; 进 而重点从时空大数据的存储管理、时空分析和领域挖掘 3 个角度对并行化现状进行了总结, 阐述了其中存在的问 题;最后指出了时空大数据研究发展趋势。
\end{abstract}

关 键 词: 时空大数据; 高性能软硬件; 并行空间分析; 数据挖掘; 进展及趋势

\section{1 引言}

随着互联网、物联网和云计算的高速发展, 数 据获取手段向多元化方向发展, 数据种类不断多样 化, 促使时空相关的数据呈现出 “爆炸式”增长的趋 势, 时空信息与大数据的融合标志着正式进人时空 大数据时代。时空大数据除具备大数据典型的 “4V”特性外, 还具备对象/事件丰富的语义特征和 时空维度动态关联特性。对时空大数据进行处理、 分析和挖掘得到蕴含的复杂特征是其核心价值所 在(李德仁, 马军等, 2015)。

在时空大数据时代中机遇与挑战并存。一方 面是时空数据量和类型的丰富, 弥补了数据缺乏的 不足, 能够在最大程度上满足各类研究的需求, 进 一步推动交叉研究的不断深人; 另一方面是面对时 空大数据时空特征的特殊性, 时空对象、事件等要 素的动态演化以及相互间的动态关联关系对数据
管理和分析带来了极大的挑战。在存储管理方面, 以往集中式存储严重依赖单机性能, 极大限制了存 储能力的可扩展性, 无法支撑海量非结构化数据低 延迟存取高并发访问。在处理分析方面, 以往串行 分析算法已无法满足海量时空数据的实时处理需 求,不能充分发挥当前新型硬件构架和并行模型/框 架的优势。在数据挖掘方面, 传统的数据挖掘算法 大多是基于常规数据集实现,推广到 $\mathrm{TB}$ 级别甚至 是 $\mathrm{PB}$ 级别数据时, 其计算效率低、扩展性能差的不 足就会显现。因此时空大数据与高性能计算/云计 算融合是必然的发展趋势,通过两者融合从而进一 步提升时空大数据的利用效率, 能更好地为研究应 用服务。

针对上述问题, 本文基于时空大数据背景, 对 现有时空大数据存储管理、时空分析和领域挖掘进 行了全面的总结和阐述。首先, 从时空大数据的概 念和起源出发, 介绍了大数据的分类和特点, 分析

收稿日期: 2018-08-31; 修订日期: 2018-10-13。

基金项目: 国家自然科学基金项目(41301411) [Foundation: National Natural Science Foundation of China, No.41301411]。

作者简介:关雪峰(1980-), 男,湖北松滋人,副教授,研究方向为高性能地理计算,E-mail: guanxuefeng@whu.edu.cn。

通讯作者: 曾宇媚(1994-), 女, 广东茂名人, 硕士研究生, 研究方向为地理信息建模, E-mail: zengyumei@whu.edu.cn。 
了时空大数据的固有特征。在此基础上总结了现 有的高性能计算平台软硬件的发展现状,包括硬件 架构、并行计算模型/框架以及各自优势对比。然 后,全面总结了现阶段时空大数据的存储管理模 式、并行分析策略和数据挖掘算法的并行化实现， 并认为并行化是支撑时空大数据进行高效分析处 理的重要手段。最后, 探讨了时空大数据时代下分 布式存储管理与并行处理分析当前发展趋势。

本文所介绍的时空大数据并行处理分析的软 硬件环境及研究应用现状见图 1。

\section{2 时空大数据}

2008 年,Nature 杂志在其发表的一篇文章“big data: Wikiomics” 中首次提出了 “大数据” 这一名词 (Waldrop, 2008)。2011 年, Science 杂志出版专刊 “dealing with data”, 探讨了如何借助宝贵的数据资 产推动人类社会向前发展(Hong et al, 2011)。2012 年, 美国针对大数据的发展热潮正式启动了一项 “大数据研究和发展计划”, 以期在从大数据中获取 知识方面有所突破。2015 年, 中国国务院也印发了
《促进大数据发展行动纲要》, 纲要中基于全球大数 据发展迅速和大数据广泛应用于各个领域的现状, 提出了中国未来在大数据的发展规划中要加快数 据共享、提高管理水平等任务。

迄今为止, 大数据科学已经发展为一门新兴的 综合性学科。对于 “大数据”, 普遍认为它是数据体 量(volume)大、数据类型(variety)多、产生速度(velocity)快和价值(value)含量高的数据集合。而时空 大数据, 则是指与时空位置相关的一类大数据, 是 时空信息与大数据的融合。日常生活中带有时间 与位置标签的数据十分常见, 人类生活中所产生的 数据约有 $80 \%$ 和时空位置有关 $(X u, 1999)$ 。2011 年,麦肯锡环球研究院 Manyika 等(2011)发布了报 告 “big data: the next frontier for innovation, competition, and productivity”, 报告提出医疗保健、零售、公 共领域、制造业和个人位置这五大类数据组成了当 前主要的大数据流, 而这些数据都具有显著的地理 编码和时间标签。因此,如何高效处理分析时空大 数据是当前学术界研究的热点问题之一。

从感知对象角度, 时空大数据可以划分为以下 2类:

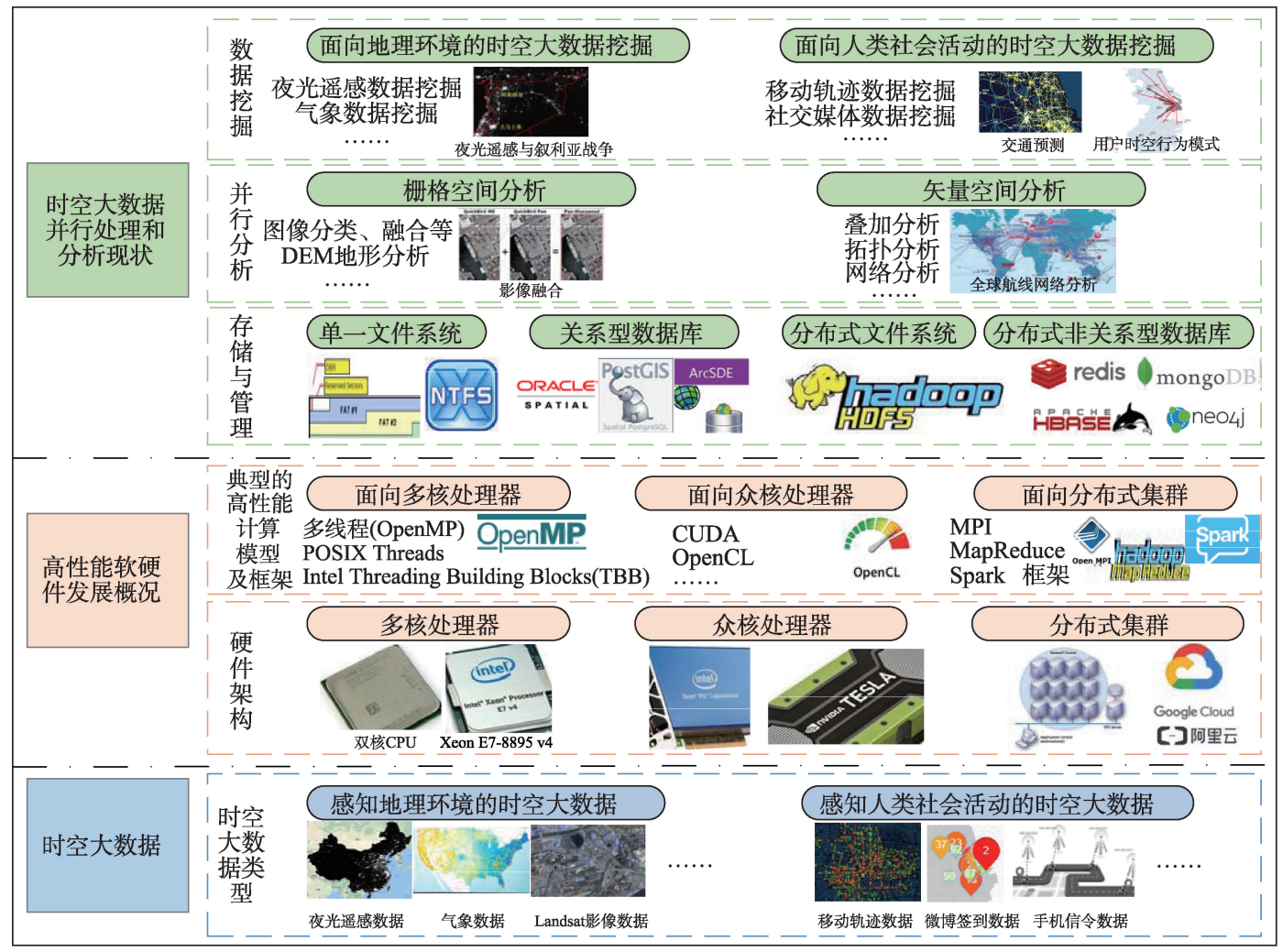

图 1 时空大数据并行处理分析的软硬件环境及研究应用现状

Fig.1 Software/hardware environment and research status quo of parallel processing and analysis on big spatiotemporal data 
(1) 感知地理环境的时空大数据

随着对地观测技术的发展, 各类遥感数据成指 数级增长并逐步积累, 成为一类典型的时空大数 据, 即“遥感大数据”。随着遥感云平台的建设, 各 类遥感数据服务与处理服务逐渐被发布出来, 地理 服务从专业走向大众,用户无需搭建专用环境就可 以方便地应用遥感大数据。2018 年 2 月, 中国科学 院正式启动 $\mathrm{A}$ 类战略性先导科技专项 “地球大数据 科学工程(CASEarth)”。它的目标是建成具有全球 影响力的、开放性的国际地球大数据科学中心, 逐 渐突破技术瓶颈, 形成资源、环境、生态等多学科领 域融合、独具特色的地球大数据云服务平台, 肩负 起国家宏观决策与重大科学发现的重任。

(2) 感知人类社会活动的时空大数据

随着互联网技术、社交媒体平台的不断发展和 进步, 人类活动每时每刻都会产生大量的时空数 据, 具有位置坐标和时间标签, 具体包括移动轨迹 数据、社交媒体数据、购物订单数据、手机信令数据 等。这些数据记录着人类的日常生活, 蕴含着人类 活动的潜在规律, 且它们正以前所未有的速度和规 模增长和累积, 亟待被合理、高效、充分地挖掘应 用。近年来, 面向人类活动的时空大数据逐渐被挖 掘、利用、生成各类智慧服务, 并渗透到人们生活的 各方面。在智慧经济方面, 企业利用数据挖掘技 术, 从客户消费的时空大数据中获取人们消费习 惯, 并划分成不同的消费群体, 从而有针对性地投 放产品, 实现精准营销; 在智慧交通方面, 通过分析 人流和车辆移动轨迹的时空大数据, 可以预测路段 的人流密度与交通状况, 从而有效改善交通拥堵现 象; 在智慧医疗方面, 通过对海量病历数据进行分 析建模, 可以了解人群疾病的时空分布规律, 从而 及时进行疾病的预防和控制。

时空大数据除了具备大数据本身所具有的海 量、多维、价值高等特征之外, 还具备对象/事件的丰 富语义特征和时空维度动态关联特性(李德仁, 马 军等, 2015), 具体包括以下 4 点:

(1) 时空大数据的要素包括对象、过程、事件 等, 且这些要素在空间、时间、语义等方面具有关联 约束关系;

(2) 时空大数据在空间和时间上具有动态演化 特性, 这些基于时空大数据要素的时空变化是可被 度量的;

(3) 时空大数据具有尺度特性, 根据比例尺大
小、采样粒度以及数据单元划分的详细程度可以建 立时空大数据的多尺度表达与分析模型;

(4) 时空大数据具备多维动态可视化特性, 实 时获取对象在不同阶段的行为特征,通过参照并映 射到三维地理空间中,可以实现动态可视化渲染效 果, 从而更好地觉察、理解和预测对象的发展。

时空大数据不断被应用于各个领域, 促进了新 的研究模式生成, 然而传统的数据存取、分析和挖 掘方法却难以支撑新的研究模式形成。日趋庞大 的数据量容易导致算法性能陷人瓶颈,用户对响应 的实时性要求越来越高, 传统集中式的数据存储管 理策略和串行时空分析算法已越来越不能满足时 空大数据高效存储和实时处理分析的需求。因此, 在分布式计算、并行计算以及云计算技术飞速发展 的背景下, 针对时空大数据的特殊性, 将高性能计 算技术应用到时空大数据的处理分析中, 实现数据 快速高效处理, 快速准确提取其中的价值信息, 是 当前时空大数据的一大研究热点。

\section{3 高性能软硬件发展概况}

近年来,计算机技术迅速发展, 在硬件方面, 计 算能力成倍增长, 硬件架构发生了巨大变化; 在软 件方面, 云计算技术的兴起,面向大数据的高性能 计算模型和处理框架不断涌现。

\section{1 硬件架构}

(1) 多核处理器

多核处理器(multi-core processor) 是在单个芯 片(die)中封装了两个或以上的独立中央处理器核 心(core), 核心间通过高速总线(bus)互联。多核处 理器通过提供不同层次的指令级并行和线程级并 行从而提高计算性能。2005 年, Intel 公司和 AMD 公司率先开发出双核处理器(彭晓明等, 2012)。 2017 年, Intel 公司发布了基于 $14 \mathrm{~nm}$ 工艺制程的 24 核处理器(Xeon E7-8894 v4), 支持 48 个线程, 频率 为 $2.4 \sim 3.4 \mathrm{GHz}$ 。

\section{(2) 众核处理器}

众核处理器(many-core processor) 是专为高度 并行处理而设计的专业多核处理器,包含大量简 单、独立的处理器内核, 并广泛用于嵌人式计算和 高性能计算中。众核处理器相对于多核处理器的 区别在于它设计的出发点是对更高程度的显式并 行进行优化, 以密集的计算线程提高吞吐量,使得 
它在处理海量结构化数据时更有优势。典型的众 核处理器有 NVIDIA GPGPU(general purpose GPU)、 Intel MIC(many integrated core)等。其中用户群最 广的是 GPGPU, 其具有高带宽、高并行性的特点, 因此在处理单指令流多数据流(SIMD)时, 面对数据 运算量远大于数据调配和传输的场景时, 其计算性 能相对于传统多核 CPU 来说有了极大的提升 (Nickolls et al, 2010)。2017 年 NVIDIA 发布了 Tesla Volta100 GPU架构, 能提供 30TFLOPS 的FP16半 精度性能, 15TFLOPS 的 FP32 单精度性能, 7.5FLOPS 的 FP64 双精度性能, 以及 120TLOPS 独 立 Tensor 操作量。Tesla Volta100 中新型流式多处 理器架构针对深度学习进行了专门优化, 在深度学 习性能上显著提升, 架构比上一代节能 $50 \%$, 且使 用 HBM2 内存, 操作更快、效率更高 (NVIDIA, 2017)。

\section{(3) 分布式集群}

分布式集群是指将相互独立的计算机节点 (node)通过高速互联网络连接起来而形成的计算集 群(cluster)。集群面对的高并发请求或计算密集任 务可以分发到所有工作节点协同完成。同时由于 节点之间通信开销成本和数据传输延迟, 因此集群 多采用粗粒度的并行任务划分策略。

随着虚拟化技术的发展与成熟, 基于云平台的 新兴计算模式一一云计算应运而生。云平台借助 虚拟化技术的伸缩性和灵活性, 提高了计算/存储/ 网络资源利用率; 通过信息服务自动化技术, 将各 类资源封装为服务交付给用户, 减少了用户使用成 本。云平台提供的服务通常可分为: 基础设施即服 务 (infrastructure as a service, IaaS)、平台即服务 (platform as a service, PaaS)、软件即服务(software as a service, SaaS)这 3 大类。与传统的集群相比, 云 平台可以将物理资源虚拟化为资源池, 灵活提供软 硬件资源, 实现对用户的按需供给, 具有资源池化、 按需服务、服务可计量等特点, 因此云平台在并行 计算中发挥着重要作用。自云计算的概念被 IBM 提出后, 得到了 Google、Microsoft、Amazon 等各大
IT 公司的重视,纷纷推出了各自的云平台, 如 Google Cloud、微软 Azure 平台、Amazon Cloud、阿 里云等。

表 1 对以上提出的硬件架构从硬件类型、计算 能力、可扩展性等角度进行了比较。

\section{2 典型的高性能计算模型及框架}

同时,对应的高性能计算模型及框架也呈现出 多元化特点, 不同的硬件结构逐步发展出与该硬件 架构相符合的高性能计算模型,如面向多核处理器 的多线程模型,包括 OpenMP, POSIX Threads 和 Intel Threading Building Blocks(TBB)等; 面向众核处 理器的 CUDA、OpenCL 等; 面向分布式集群的 MPI、MapReduce 和 Spark 框架等。

\subsection{1 面向多核处理器的并行计算模型}

多线程模型是一种基于多线程并发执行(multithreading)来提升算法处理能力或效率的并行程序 开发模型。在一个多线程程序中,一个主进程通常 创建多个工作线程,每个工作线程并行执行不同的 任务,共享主进程中的全部系统资源。其中具有代 表性的多线程模型是 OpenMP, 它支持 $\mathrm{C} 、 \mathrm{C}++$ 和 Fortran 语言, 可以实现任务并行和数据并行 (Dagum et al, 1998)。OpenMP不仅可以利用编译指令 自动进行任务分解, 允许渐进式并行化, 而且对原串 行代码不需要进行重大改变, 具有良好的可移植性。 然而 OpenMP 只能在共享内存的多核/多处理器平 台上高效运行, 可扩展性受到存储器架构的限制。

\subsection{2 面向众核处理器的并行计算模型}

(1) CUDA(compute unified device architecture, 统 一计算设备架构)。CUDA 是 2007 年 NVIDIA 公司 推出的运行在本公司各种型号 GPU上的一种通用 并行计算架构(Garland et al, 2008), 最初基于 C 语言 环境, 如今可支持 $\mathrm{C} 、 \mathrm{C}++$ 和 Fortran 等编程语言。 CUDA 能够为用户提供统一的开发框架和编程模 型, 辅助用户快速构建高性能应用程序并充分发挥 GPU 的优势, 从而极大提高了通用计算能力。2017 年发布了专为 Volta GPU 而构建的 CUDA 9, 更快的 GPU 函数库, 基于协作组(cooperative groups)新的

表 1 硬件架构对比

Tab.1 Comparison of different hardware architectures

\begin{tabular}{ccccl}
\hline 硬件架构 & \multicolumn{1}{c}{ 类型 } & 计算能力 & 可扩展性 & \multicolumn{1}{c}{ 优点 } \\
\hline 多核处理器 & Intel Xeon CPU等 & 中等 & 中等 & 多个内核同时处理,可实现多任务处理和计算 \\
众核处理器 & GPGPU、MIC等 & 较强 & 中等 & 高度并行性,计算速度快,数据吞吐能力高 \\
分布式集群 & 云平台等 & 强 & 高 & 节点松散耦合,节点内部可以集成多/众核处理器,扩展性非常强 \\
\hline
\end{tabular}


编程模型, 进一步加快了应用程序的编译速度。 2018 年发布的 CUDA 9.2 具有更低的内核启动延 迟, 启动 CUDA 内核的速度比 CUDA 9 快 2 倍。

(2) OpenCL。OpenCL 为异构计算提出的统一 编程标准, 在由 CPU、GPU、FPGA 或其他处理器等 组成的异构平台中, OpenCL 可以提供基于任务划 分和基于数据划分的并行计算机制 (Javier et al, 2012)。OpenCL 支持跨平台和硬件体系结构编程 的特点,使得它在面对异构计算时具有强大的优势。 目前OpenCL 2.2 版本通过将OpenCL C++内核语言 引人到核心规范中, 从而显著提高并行计算的效率。

\subsection{3 面向分布式集群的并行计算模型及框架}

(1) MPI。MPI 是一个基于消息传递的并行计 算应用程序接口(Dinan et al, 2016), 主要应用于分 布式集群上, 支持点对点和广播 2 种通信方式, 典型 的开源实现有 OpenMPI、MPICH 等。MPI 可移植性 强, 能同时应用于分布式内存/共享内存的处理平 台。在分布式集群上通常采用混合编程模型 (Hybird), 同时结合了 OpenMP 和 MPI 两者的优点, 基 于 OpenMP 实现线程级并行, 同时在节点间基于 MPI 实现任务分配和消息传递, 以实现线程和进程 2 个层次的并行计算(赵永华等, 2005)。目前 MPI 仍然是当今大型计算密集型应用主要使用的并行 计算模型。

(2) MapReduce。Google 公布的关于 Google
File System(Ghemawat et al, 2003)、MapReduce(Dean et al, 2004)和BigTable(Chang et al, 2008)的 3 篇技术 论文, 奠定了当前云计算发展的重要基础。其中 MapReduce 并行开发模型面向大规模数据集的并 行处理,能够实现计算任务的自动并行和调度, 因 其具有简单适用的特点而被广泛应用(李建江等, 2011)。MapReduce 模型把计算过程抽象为 2 个阶 段, 即 Map 和 Reduce, 用户通过实现 map(映射)和 reduce(规约) 2 个函数,从而实现分布式计算。结合 MapReduce 并行框架可实现海量的并行计算任务 自动并发执行, 同时隐藏底层实现细节, 大大降低 编程难度(杜江等, 2015)。

(3) Spark。Spark 是 UC Berkeley 大学 AMP 实 验室在 2009 年提出的一个开源通用并行计算框架, 以支持海量数据集的并行处理 (Zaharia et al, 2016)。Spark提供了一个基于集群的分布式内存抽 象, 即弹性分布式数据集(RDD), RDD 作为一个可 并行操作、有容错机制的数据集合, 提供了统一的 分布式共享内存。Spark使用了内存计算技术, 减 少磁盘 $I / O$, 允许多次循环访问内存数据集, 有助于 实现迭代算法, 从而进行交互性或探索性数据分 析; 同时容错性高, 保证了分布式应用的正确执 行。因此 Spark 在大数据处理领域中发挥着越来越 重要的作用,但也同时存在内存消耗大的问题。

表 2 从出现时间、支持硬件、并行粒度、内存访

表 2 典型的高性能计算模型及框架的对比

Tab.2 Comparison of high-performance computing models and frameworks

\begin{tabular}{|c|c|c|c|c|c|c|c|c|}
\hline $\begin{array}{c}\text { 并行计算模型 } \\
\text { 分类 } \\
\end{array}$ & $\begin{array}{l}\text { 并行计算 } \\
\text { 模型/框架 }\end{array}$ & $\begin{array}{l}\text { 出现 } \\
\text { 时间 }\end{array}$ & 支持硬件 & 并行粒度 & $\begin{array}{c}\text { 内存访问 } \\
\text { 模型 }\end{array}$ & 性能优势 & 不足 & 适用场景 \\
\hline $\begin{array}{l}\text { 面向多核处理 } \\
\text { 器的并行计算 } \\
\text { 模型 }\end{array}$ & $\begin{array}{l}\text { 多线程(以 } \\
\text { OpenMP为例) }\end{array}$ & 1997 & CPU、MIC & 细粒度 & 共享内存 & $\begin{array}{l}\text { 实现简单, 并行效 } \\
\text { 率高, 可移植性强 }\end{array}$ & $\begin{array}{l}\text { 只适用 SMP 等并行 } \\
\text { 环境,不适合集群, } \\
\text { 扩展性较差 }\end{array}$ & $\begin{array}{l}\text { 简单处理算法级 } \\
\text { 的并行与优化 }\end{array}$ \\
\hline \multirow[t]{2}{*}{$\begin{array}{l}\text { 面向众核处理 } \\
\text { 器的并行计算 } \\
\text { 模型 }\end{array}$} & CUDA & 2007 & GPU & 细粒度 & 共享内存 & $\begin{array}{l}\text { 基于 } \mathrm{C} \text { 语言, 有强 } \\
\text { 大的并行浮点计 } \\
\text { 算能力 }\end{array}$ & $\begin{array}{l}\text { 仅能在 GPU 硬件上 } \\
\text { 使用,通用性较弱 }\end{array}$ & $\begin{array}{l}\text { 适合大规模数据 } \\
\text { 密集型并行计算 }\end{array}$ \\
\hline & OpenCL & 2008 & $\begin{array}{l}\text { CPU、GPU、 } \\
\text { FPGA 等 }\end{array}$ & 细粒度 & 共享内存 & $\begin{array}{l}\text { 支持跨平台和硬 } \\
\text { 件体系结构编程, } \\
\text { 可移植性强 }\end{array}$ & $\begin{array}{l}\text { 开发环境完善性较 } \\
\text { 差,API设计缺乏一 } \\
\text { 致性 }\end{array}$ & $\begin{array}{l}\text { 适合异构平台计 } \\
\text { 算 }\end{array}$ \\
\hline \multirow[t]{3}{*}{$\begin{array}{l}\text { 面向分布式集 } \\
\text { 群的并行计算 } \\
\text { 模型 }\end{array}$} & MPI & 1992 & CPU 、MIC & $\begin{array}{l}\text { 细/粗 } \\
\text { 粒度 }\end{array}$ & 分布式内存 & $\begin{array}{l}\text { 适用于集群环境, } \\
\text { 并行算法扩展性 } \\
\text { 强 }\end{array}$ &  & 计算密集型应用 \\
\hline & MapReduce & 2004 & $\mathrm{CPU}$ & 粗粒度 & 分布式内存 & $\begin{array}{l}\text { 编程实现简单, 容 } \\
\text { 错性好, 计算能力 } \\
\text { 较强 }\end{array}$ & $\begin{array}{l}\text { 模型单一, 适合批处 } \\
\text { 理模式,但中间结果 } \\
\text { 要写人磁盘, 不适合 } \\
\text { 迭代运算 }\end{array}$ & $\begin{array}{l}\text { 数据密集型应用、 } \\
\text { 分布式数据处理 }\end{array}$ \\
\hline & Spark & 2010 & $\mathrm{CPU}$ & 粗粒度 & 分布式内存 & $\begin{array}{l}\text { 丰富的 API,减少 } \\
\text { 磁盘 I/O,适合迭 } \\
\text { 代算法 }\end{array}$ & 内存消耗较大 & $\begin{array}{l}\text { 适合图计算、迭代 } \\
\text { 计算和交互式数 } \\
\text { 据分析 }\end{array}$ \\
\hline
\end{tabular}


问模型、性能优势、不足以及适用场景等方面对上 面列举的高性能计算模型及框架进行了分析比较。

\section{4 时空大数据并行处理与分析进展}

\section{1 时空大数据存储与管理}

时空数据的存储管理方式伴随着计算机技术 的发展而不断变化和更新, 在每一阶段都受到当前 计算机软硬件水平、数据规模特征、实际应用需求 等因素的影响, 使得数据存储管理模式不断演化, 从传统的集中文件存储/空间数据库, 发展到以 Hadoop HDFS 为代表的分布式文件系统管理, 再到当 前的分布式NoSQL 非关系型数据库。

\subsection{1 集中式存储}

传统的集中式数据存储将存储与应用分离, 上 层应用通过中间件连接, 访问数据时需要通过网络 对集中存储的数据进行快速访问。集中式存储中 主要有基于文件和基于数据库这 2 种管理模式。

(1) 基于文件的管理模式。通常是基于单一文 件系统(如 FAT、NFS 等) 将数据以 “文件” 的形式存 储在磁盘等可以直接访问的介质中, 并提供了一系 列存取、索引、更新等操作。这种管理模式存取高 效、操作简单, 但存储空间扩展性、数据结构兼容 性、数据安全性都很差, 且存储能力受到存储介质 性能的极大影响。

(2) 基于数据库的管理模式。这是当前集中式 存储管理的主流模式, 利用成熟数据库技术来组 织、存储和管理各类数据。传统的关系型数据库在 处理结构化数据中有着很大优势, 在容量上关系型 数据库可采用分区技术对上亿级别的数据进行存 储管理以提高访问性能; 在并发访问能力上, 关系 型数据库相对于传统的文件系统来说, 它能够从容 地应对多用户的高并发访问场景 (李绍俊等, 2017)。20世纪 90 年代, 基于关系型数据库的空间 数据存储管理作为当时的主流应用模式, 催生了众 多成熟的空间数据库, 常见的关系型空间数据库有 Oracle Spatial、PostGIS、ArcSDE 等。空间数据引擎 为用户和空间数据库之间提供了一个开放性接 口。基于空间数据引擎和关系型数据库在应用程 度中结合的紧密程度, 提出了内置、两层结构和三 层结构这 3 种空间数据引擎结构。内置模式是直接 在关系型数据库内核新建一个空间扩展模块, 模块 提供了针对空间数据的一系列操作, 典型的有 Ora- cle Spatial、PostGIS 等。两层结构模式是可以直接 访问空间数据访问客户端和数据库服务端, 典型的 有 SuperMap SDX + 。三层结构模式在客户端和数 据库服务端中间新增设空间数据应用服务器, 并通 过中间的服务层来对客户端的数据访问请求进行 统一处理和响应, 典型的有 ESRI ArcSDE。

集中存储模式能够在一定程度上解决海量空 间数据存储和管理所面临的问题。然而, 在大数据 时代下随着数据获取途径多样化、快速化发展, 数 据逐渐呈现出非结构化、高时效性的特点, 因此传 统集中、单一的数据存储方式越来越不能满足大数 据时代下非结构化数据存储和管理的实际应用需 求, 同时对多用户高并发访问能力提出了更高的 要求。

\subsection{2 分布式文件系统}

2003 年, Google 研发出了谷歌文件系统 GFS (Google file system)(Ghemawat et al, 2003)。GFS 是 专门针对 Google 计算机集群为 Google 的页面搜索 数据存储进行优化的一个可扩展的分布式文件系 统, 集群中节点由众多廉价的服务器组成, 主要面 向大文件和读操作较多的场景。GFS 中数据分块 存储, 采用了 master-slave 结构对海量数据按照一定 的顺序进行高效存储 (孟小峰等, 2013)。对应开源 的实现有 Hadoop HDFS(HDFS, 2012)、Facebook 专 门针对海量小文件推出的 Haystack(Beaver et al, 2010)等。

HDFS 主要是面向大文件设计的,基于分布式 集群架构实现结构化时空大数据的存储和管理, 具 有较好的可扩展性和容错性。王凯等(2015)从 Hadoop 不支持传统空间数据的问题出发, 提出了一种 针对矢量空间数据的存储格式, 并在 Hadoop 环境 下对 GIS 大数据进行处理, 有效提高了 GIS 大数据 的计算效率; 尹芳等(2013)对 Key/Value 键值对数据 模型进行了分析, 为矢量数据能够在 HDFS 中进行 高效存储而设计出了一种符合 GeoJSON 地理数据 编码的矢量数据 Key/Value 文本格式, 通过 HDFS 的 数据自动分块功能将海量矢量数据自动分割成大 量的小数据块, 分别存储到不同节点上, 以实现海 量矢量数据的分布式高效存储。

分布式文件系统支持数据分块存储, 具有高扩 展性和高可靠性, 然而随着用户访问数量激增, 分 布式文件系统无法提供统一的访问接口以及高效 的数据查询检索能力。 


\subsection{3 分布式非关系型数据库}

基于分布式数据库实现半结构化或非结构化 时空大数据的存储与管理是当前数据库的重要发 展趋势。NoSQL 是指非关系型、分布式、不保证遵 循关系型数据库 ACID 原则的数据库的统称 (NoSQL, 2009), 可为时空大数据提供低成本、高扩 展性、高通量 $\mathrm{I} / \mathrm{O}$ 平台, 从而解决多用户高并发场景 下海量、快速增长的半结构化和非结构化数据的高 效、灵活的存储和管理问题(Ghemawat et al, 2003; 马林, 2009)。目前主流的开源 NoSQL 数据库主要 可分为 4 类(Hecht et al, 2012): (1)面向 Key-Value 存 储,如Redis、Berkeley DB、MemcacheDB等;2面向 列存储, 如 HBase、Cassandra等; (3)面向文档存储, 如 MongoDB、CouchDB 等; (4)面向图存储, 如 Neo4J、FlockDB 等。分布式非关系型数据库提供 了分布式 $I / O$ 、索引结构、查询执行和优化等一系列 高效管理操作。具体分类如表 3 所示。

Redis 作为高性能面向 Key-Value 存储的数据 库, 数据吞吐量大, 可实现高速读写, 适合数据读写 操作多的应用场景。然而 Redis 基于内存存储的特 点对内存的消耗过大, 扩展性较差。在 Redis 的应 用中, 张景云等(2013)为了提高元数据信息和矢量 空间数据几何与属性信息的存储效率,采用了基于 Redis 实现矢量空间数据按照库、集、层、要素 4 级结 构进行存储的层次组织, 提高了矢量空间数据的管 理组织效率; 间密巧等(2017)基于 Redis 数据库为 海量轨迹数据设计了针对轨迹数据特性的存储方 案及数据存储结构, 从而提高了在线平台的轨迹数 据实时存储效率。

HBase 分布式数据库支持半结构化和非结构化 时空数据存储与管理, 满足海量数据存取和时空查 询的应用需求。在矢量数据存储方面, 郑坤等 (2015)针对矢量空间数据设计了基于 HBase 的高效
存储模型, 实现了对矢量空间数据的直接存取和展 示, 提高了矢量空间数据的存储效率; 在栅格数据 存储方面, 张晓兵(2016)基于 HBase 的高扩展性设 计了一个弹性可视化遥感影像存储系统, 解决了海 量影像瓦片数据的快速存储问题。

MongoDB 提供了多种类型的空间索引,包括 B-tree 索引、GeoHash 索引等, 从而更好地支持海 量数据的分片存储。在矢量数据存储方面,雷德龙 等(2014)基于 MongoDB 和三层云存储架构的优势, 为海量矢量空间数据的高效存储管理与处理分析 设计出了 VectorDB; 在栅格数据存储方面, 田帅 (2013)、张飞龙(2016)都将 MongoDB 和分布式文件 系统结合起来设计了海量遥感数据存储管理系统, 其中采用基于 MongoDB 的高性能存储架构对遥感 影像元数据进行高效存取, 针对遥感影像文件数据 则采用了分布式文件系统进行存储, 此系统的混合 存储策略实现了海量遥感影像数据的高效存取并 提高了存储资源的利用率。

Neo4J 是一个面向图操作的高性能、高可靠性 的开源图形数据库。马义松等(2016)基于 Neo4J构 建了一个电网的全景数据库, 基于该数据库对电网 中具有分散、隔离特性的电力大数据进行了有序整 合。廖理(2015)针对关系型数据库存储效率低、扩 展性差等特点提出了一种基于 $\mathrm{Neo} 4 \mathrm{~J}$ 的时空数据存 储模型, 该模型能够有效地将空间、时间和属性信 息整合起来进行建模和存储, 提高时空数据存储 效率。

分布式非关系型数据库侧重于提高半结构化 和非结构化数据的存储效率, 可提供优于关系型数 据库的低成本、高可扩展性、高并发能力和高通量 $\mathrm{I} / \mathrm{O}$ 的海量数据存储平台。然而, 其在互联网领域 的应用模式与传统的 GIS 领域还存在差异, 使得 NoSQL 数据库在数据操作方式、时空索引支持、查

表 3 目前主流的 NoSQL 数据库分类

Tab.3 Classification of classic NoSQL database

\begin{tabular}{|c|c|c|c|c|c|c|c|}
\hline 分类 & 数据库 & 支持平台 & 存储性能 & 灵活性 & 复杂性 & 优势 & 不足 \\
\hline $\begin{array}{l}\text { 面向 } \\
\text { Key-Value }\end{array}$ & Redis, MemcacheDB等 & Linux & 高 & 高 & 无 & $\begin{array}{l}\text { 内存数据库、可实 } \\
\text { 现高速读写 }\end{array}$ & $\begin{array}{l}\text { 内存消耗较大, } \\
\text { 扩展性较差 }\end{array}$ \\
\hline 面向列 & HBase, Cassandra等 & Linux, Windows & 高 & 中等 & 低 & $\begin{array}{l}\text { 数据压缩率高, 支 } \\
\text { 持快速的 OLAP }\end{array}$ & $\begin{array}{l}\text { 没有原生的二级 } \\
\text { 索引 }\end{array}$ \\
\hline 面向文档 & MongoDB, CouchDB 等 & $\begin{array}{l}\text { Linux, Mac, } \\
\text { Windows }\end{array}$ & 高 & 高 & 低 & $\begin{array}{l}\text { 面 向 Document, 支 } \\
\text { 持空间数据管理 }\end{array}$ & $\begin{array}{l}\text { 不支持事务操作, } \\
\text { 占用空间过大 }\end{array}$ \\
\hline 面向图 & Neo4J, FlockDB 等 & $\begin{array}{l}\text { Linux, Mac, } \\
\text { Windows }\end{array}$ & 可变 & 高 & 高 & $\begin{array}{l}\text { 高精度的图算法, } \\
\text { 图查询迅速 }\end{array}$ & $\begin{array}{l}\text { 没有分片存储机 } \\
\text { 制, 图数据结构写 } \\
\text { 人性能较差 }\end{array}$ \\
\hline
\end{tabular}


询访问模式等方面仍存在较大的局限性。

\section{2 时空大数据并行分析}

时空大数据因其自身特点及存储模式的变化, 使得传统的串行分析算法存在很大的局限性, 不能 充分利用和发挥当前新型硬件构架(单机多核、集 群、集群/众核混合等)资源的优势, 难以满足实际应 用的规模与高效需求, 因此时空大数据的并行分析 已成为当前研究的热点。

传统 GIS 数据, 主要以结构化的栅格影像和半 结构化的矢量要素形式存储。对于这类空间数据 的分析, 经过长期的发展目前已形成较为成熟的串 行算法库, 并且对应的 GIS 软件平台非常成熟, 如 ArcGIS、SuperMap、GRASS 等; 然而这些串行算法 及平台随着 GIS 数据量和计算规模的逐渐增大, 难 以满足实际的应用需求, 因此并行空间分析算法受 到越来越多的关注。作为 GIS 的 2 大基础数据结 构, 栅格和矢量具有不同的特征与优势, 对于地理 要素的表达形式不同,分析方法不同,因此栅格空 间分析与矢量空间分析的并行化策略也各有侧重。

(1) 对于栅格空间分析来说, 每一个像元上的 计算形式相同且相对独立, 单个像元计算任务复杂 度低, 因此多采取数据并行的策略, 即在数据分块 的基础上利用并行计算技术进行处理分析。过去, 栅格空间分析往往采取 CPU 并行的方法, 但随着 GPU 通用计算能力的发展以及在数据并行上的良 好适应性,越来越多的栅格分析方法开始采取基于 GPU 的并行化策略。在图像分类、融合和滤波等方 面, 杨靖宇等(2010)从 GPU 的并行性和流式编程模 型出发, 为图像的高效处理分析设计了一种流水线 并行处理模式, 实现了影像光谱角匹配算法的并行 化; 卢俊等(2009)充分发挥 GPU可编程渲染器和并 行处理数据的优势, 提出了基于 GPU 的遥感影像 IHS 融合算法, 其将 IHS 算法映射到 GPU 的流式计 算中, 结果显示该算法的处理速度明显优于传统基 于 CPU 的算法; 杨洪余等(2017)利用 CUDA 编程模 型的特性, 提出了面向 CPU/GPU 异构环境的图像 协同并行处理模型, 结果显示该模型在灰度图像处 理中处理速度得到了较大提升。在 DEM 地形分析 方面, Do 等(2011)为了提取 DEM 的排水网络以获 取全局的流量累积, 从而提出了一种并行生成树方 法对集水盆地进行分层统计, 结果显示该方法无需 完整的 DEM, 分析效率高, 扩展性强; Qin 等(2012) 提出了一种在 GPU 上兼容 CUDA 计算流量累积的
并行方法, 对 DEM 数据预处理在 GPU 上进行了并 行化实现,针对递归 MFD算法的并行化提出了基 于图论的并行化策略,结果显示该策略在流量累积 计算中处理速度得到了很大的提升。然而,这些并 行化方法都是针对单个空间分析算法的具体实现， 属于细粒度的线程级并行。与之相对的粗粒度并 行编程模型如 MPI/MapReduce 则是通过栅格数据 分割实现分布式计算, 有效利用集群资源。Xu等 (2014)提出了一种基于 MPI和 MapReduce 并行计算 模式的栅格加权 Voronoi 图生成算法, 该算法显著 提高了利用大规模栅格数据生成 Voronoi 图的效 率, 并在城市公共绿地规划和最优路径规划中得到 验证; 程果等(2012)基于 MPI并行计算模型提出了 栅格地形分析中坡度坡向计算的并行化方法,有效 降低了数据通信成本, 充分利用了并行计算资源。 更进一步, 为了提高并行程序的可移植性, 在栅格 分析的通用并行框架研究方面, Qin等(2014)提出了 一种面向栅格空间分析的并行框架 PaRGO, 该框架 能够兼容OpenMP、CUDA以及MPI。

(2) 对于矢量空间分析来说, 由于矢量数据是 不定长结构,且实体之间存在着复杂的空间关系， 尤其是空间实体之间的拓扑关系导致传统基于任 务的数据划分方法会造成数据量存在很大差异, 从 而导致并行任务负载不均衡、数据通信成本高等种 种问题。因此,目前的研究大体上可以分成 3 个方 向: (1)从矢量数据划分策略人手, 将其转化为数据 并行, 涉及索引构建、负载平衡、通信调度等。贾婷 等(2010)从空间数据的拓扑特征出发,在矢量空间 数据划分策略中采用了 Hilbert空间填充曲线,并改 进了 $\mathrm{K}$ 均值聚类算法对矢量空间数据进行均衡划 分; 刘润涛等(2009)从矢量空间数据的查询和索引 结构出发,对 $\mathrm{K}$ 均值算法中基于 $\mathrm{R}$ 树和四叉树的空 间索引结构进行优化, 提高了矢量空间数据的查询 和索引效率。(2)从矢量空间分析并行化算法本身 人手,算法主要包括空间叠加分析、空间关系运算 和网络分析等。在叠加分析方面,一般采用管道叠 加、数据并行叠加和块式叠加这 3 种并行策略 (Wilson, 1994; Langendoen, 1995), Qatawneh 等(2009)在 管道网络配置中并行实施 Liang-Barsky 限幅算法, 实现了大规模的多边形裁剪。在空间关系的运算 方面,朱效民等(2013)基于线段求交和点面叠加这 2 个基础空间分析算法, 采用OpenMP 进行了并行化 实现,该方法利用数据排序及 OpenMP 的动态调度 
特点优化了并行算法的内存管理, 从而提高了并行 算法的加速比; 在网络分析方面, 主要通过网络复 制及网络分割实现并行化分析(隽志才等, 2006; 卢 照等, 2010); 在大规模网络路径分析中, 网络分割 策略求解最短路径的性能更好,Lanthier 等(2003)给 出了三角不规则网络上欧氏和加权度量的最短路 径算法的并行实现; 在空间插值方面, 王鸿琰等 (2017) 以薄板样条函数插值为例, 提出了一种 CPU/ GPU 协同并行的插值算法以加速海量 LiDAR 点云 生成 DEM, 并行算法取得了 19.6 倍的加速比; Wu 等(2011)面向亿级的激光雷达点数提出了基于多核 平台的 Delaunay 三角网并行构建算法 ParaStream, 提高了数据吞吐量和降低了内存占用量。(3)大 规模集群的并行编程框架人手, 主要是指基于 MapReduce/Spark 应用于海量非结构化或半结构化数 据上的处理分析特点和优势, 使得其在矢量空间分 析并行化过程中被广泛应用。王凯等(2015)在 Hadoop 环境下对 GIS 大数据设计了一种更高效的并 行处理模型, 基于该模型进一步增强了 Hadoop 的 空间计算能力; 2013 年, ESRI ArcGIS 10.2 对 Hadoop 上的空间运算类库进行扩展, 将 GIS 数据与 Hadoop 分析相结合, 推出 “GIS Tools for Hadoop”为 空间大数据处理提供了基于 Hadoop 的并行分析环 境, 该框架充分利用了 MapReduce 进行并行数据计 算, 进一步提高了 GIS 空间计算效率和能力。未 来,ESRI的分布式并行计算会将重心从MapReduce 转移到 Spark 上, 进一步挖掘 Spark 的优势。Cheng 等(2016)基于超图模型, 研究了海量空间数据分布 式处理的调度策略的研究, 并对 $\mathrm{I} / \mathrm{O}$ 进行了优化, 提 升了海量数据的处理效率。

总的来说, GIS 数据的空间分析并行化策略已 日趋成熟, 研究学者们对栅格和矢量这两类数据的 空间分析算法并行化策略进行了大量的研究, 卓有 成效。然而, 目前大部分的策略是针对单一算法的 数据并行, 未形成通用、成熟的空间分析算法并行 框架, 扩展性不强。因此, 开发出自适应多种硬件 平台、同时支持矢量/栅格空间分析功能的 GIS 并行 框架是目前迫切需要解决的问题。

\section{3 时空大数据挖掘}

时空数据分析, 侧重于利用已有数据集进行数 据转换、处理以及简单的信息提取等操作, 为用户 决策提供依据。而相较于时空数据分析, 时空数据 挖掘综合了人工智能、机器学习、领域知识等交叉
方法, 旨在从大规模数据集中发现高层次的模式和 规律, 揭示时空数据中具有丰富价值的知识, 为对 象的时空行为模式和内在规律探索提供支撑。目 前, 时空大数据挖掘作为一个新兴的研究方法, 已 在众多领域得到广泛应用,如交通监管、犯罪预测、 环境监测、社交网络等。

传统的数据挖掘算法多基于小型数据集, 研究 更关注模型精度和规律识别能力, 往往忽略模型执 行效率和数据吞吐能力。然而, 面对 $\mathrm{TB}$ 级别甚至 是 $\mathrm{PB}$ 级别的非结构化海量数据时, 基于单机处理 的传统数据挖掘算法, 数据处理效率低, 其计算能 力难以满足时空大数据挖掘的需求, 因此如何在时 空大数据时代下进行高效的数据挖掘是目前面临 的一大难题。云计算技术的出现和迅速发展,为研 究大数据时代下的高性能数据挖掘方法提供了更 高的可能性, 利用云平台动态资源分配以及并行计 算的能力,将传统的数据挖掘串行算法转化成并行 算法, 并有效移植到云平台中, 从而实现数据挖掘 的并行化和高效化。

(1) 面向地理环境的时空大数据挖掘

随着遥感成像方式的多样化以及遥感数据获 取能力的增强, 遥感领域进人了 “大数据” 时代。然 而现有的遥感影像分析和处理技术与 “遥感大数 据”之间的不匹配, 难以采用已有技术对遥感大数 据进行充分挖掘, 因此基于遥感大数据的特点进一 步促进数据挖掘技术的发展和优化是目前遥感领 域的前沿问题之一。

遥感大数据挖掘应用广泛, 不仅可以发现不同 尺度下的地理空间演变规律, 还被应用于反映人类 社会活动的社会经济估算、环境污染监测、城市化 监测等方面。 $\mathrm{Li}$ 等(2014)基于叙利亚 2008-2014年 间的夜间灯光数据进行分析, 结果显示各区域内流 离失所者的数量与夜间灯光损失呈线性相关, 相关 系数达 0.5 以上, 说明了夜光遥感数据分析能对叙 利亚战争危机进行有效监测。Zhang 等(2018)基于 Gaofen-1 160 m 空间分辨率的 AOD 数据、大气模式 模拟数据, 提出了嵌套线性混合效应模型, 预测了 超高分辨率的 $\mathrm{PM}_{2.5}$ 日均浓度。Zhao 等(2018)利用 东南亚国家 1992-2013 年间的 DMSP/OLS 夜间灯 光数据, 基于像素级夜间灯光亮度与东南亚城市的 相应空间梯度之间的二次关系，划分成低、中、高、 极高 4 种类型的夜间照明区域, 对城市化发展进行 动态分析, 结果显示, 不同夜间照明区域之间的过 
渡模式描绘出城市化发展的不同模式。除此上述 典型案例外, 李德仁等(2013)和李德仁等(2014)提 出了基于 “OpenRS Cloud” 的遥感大数据挖掘平台, 充分利用分布式计算的优势对多源、海量遥感数据 进行存储、分析等,实现了遥感大数据的高效存取, 进而利用机器学习、人工神经网络、云模型等方法 逐步探索遥感大数据间蕴藏的内在联系及知识, 进 一步实现从遥感数据到知识的转变。

(2) 面向人类社会活动的时空大数据挖掘

面向人类社会活动的时空大数据挖掘中最具 代表性有移动轨迹大数据挖掘和社交媒体大数据 挖掘。

移动轨迹数据是在时空维度下对运动物体的 移动过程进行数据收集所获得的数据信息, 具有规 模大、种类多、变化快、价值高但密度低、精度低等 特点, 包括浮动车轨迹数据、人类出行轨迹数据 等。这些数据刻画了个体和群体在时空环境下的 时空动态性, 蕴含着人类、车辆等对象的移动模式 和行为特征,对城市空间规划、交通状况预测、个性 化服务等应用具有重要的价值。现阶段,云计算等 新兴数据处理方法为移动轨迹大数据的高效分析、 深度挖掘提供了新的发展方向。在时空关联规则 挖掘方面, Chester 等(2011)采用了 MapReduce 并行 计算模型对 FP-Growth算法进行了并行化实现, 同 时还优化了数据挖掘并行策略, 从而提高了时空挖 掘的处理速度和能力。谢欢(2015)基于 Spark 框架 实现了传统协同过滤算法和时空关联规则 FPGrowth算法的并行化优化, 并行化后的算法进一步 提高了数据处理效率和数据挖掘能力。夏大文 (2016)基于 Hadoop 中的 MapReduce 并行处理模型 搭建了面向移动轨迹大数据的分布式计算平台, 提 出了基于 MapReduce 的并行频繁模式增长算法 MR-PFPh 和交通流量预测分布式建模通用框架 MF-TFF, 能有效挖掘出交通时空特征、实时交通流 量等数据背后蕴藏的有价值信息。

社交媒体与地理位置服务的结合与应用, 产生 了包含丰富的空间、时间和语义等信息的海量社交 媒体数据, 如微博签到数据、点评数据等, 具有数据 量大、产生速度快、现势性高但数据质量参差不齐 等特点。由于社交媒体数据与人类生活息息相关, 社交媒体大数据挖掘受到越来越多的关注, 探索用 户的时空行为模式成为研究热点之一。罗俊(2016) 提出了基于 LFM 的局部敏感哈希的 MapReduce 并
行化 k-means 聚类的协同过滤算法,并应用于用户 个性化推荐系统中, 从而减少了传统 k-means 算法 处理海量高维数据时的计算量和迭代次数, 提高了 用户个性化推荐系统的可拓展性和实时性。廉捷 (2013)针对网络爬虫数据大、效率低等问题,采用了 Fetch-List索引模式对网络爬虫进行并行化优化,使 得优化后的网络爬虫系统能同时多线程获取感兴 趣的数据, 减少了数据获取时间; 同时基于爬虫获 取的数据采用 SVM 模型权重优化算法进行并行化 处理和分析,提高了利用网络数据预测信息的准确 度,用于预测信息传播方向。

时空大数据挖掘在众多领域中得到广泛应用, 基于云计算框架的分布式数据挖掘算法研究已成为 热点。然而,数据挖掘算法并行化仍然受算法自身 的限制较多,如挖掘任务数据使用模式、任务之间的 相关性、任务执行流程等。而且,如今人工智能算 法发展如火如茶, 给大数据挖掘算法提供了新的模 型和方法。人工智能算法多为 “黑箱” 模型, 将底层 数据挖掘过程隐藏起来, 使大数据挖掘更方便, 但 也同时使得挖掘算法扩展性变差、并行难度加大。

\section{5 当前研究发展趋势}

综上, 正是由于时空大数据相较过去在存储管 理、处理分析、智能挖掘等方面日益增长的性能需 求, 时空大数据并行处理、分析、挖掘在面临上述问 题的同时,也涌现了新的发展趋势。这些并行化发 展趋势可总结为以下 3 点:

\section{(1) 异构计算逐渐成为计算主流}

大数据时代计算需求的多元化促进了 CPU/ GPU 异构计算的快速发展(卢风顺等, 2011)。由于 CPU 和 GPU 在硬件设备和计算方式上具有显著差 异, CPU 主要是面向复杂多任务逻辑, GPU 则具有 更高的通用计算能力, 更适合用于海量结构化数据 的并行化处理和分析。因此,异构计算平台具有很 大的发展潜力, 综合 CPU 和 GPU 两者的优势制定 一个高效合理的协同方式,保证 CPU 和 GPU 之间 的计算负载均衡, 降低两者由于数据处理方式不同 而带来的额外成本, 促进双方资源的合理配置, 使 异构计算平台在时空数据处理分析和挖掘中发挥 出最佳性能。

(2) 时空计算与时空大数据存储融合一体化 时空大数据处理面临的挑战本质上是计算平 
台的处理能力与大数据处理的问题规模之间的矛 盾。传统以计算为中心的数据处理模式将存储与 计算相互分离, 在实际处理操作中常常面临着内存 容量有限、 $\mathrm{I} / \mathrm{O}$ 压力过大等难题, 使得数据处理体系 难以达到最佳性能。目前在时空大数据环境下, 面 对数据量成指数级别增长的趋势, 研究学者已经认 识到数据的存储、传输成为制约算法/模型性能提升 的另一个关键因素, 因此将计算与存储相互融合是 计算技术发展的重要方向, 具有良好的发展前景 (殷进勇等, 2015)。

(3) 面向时空智能分析框架的成熟化

随着云计算框架的不断发展更新, 分布式数据 挖掘算法研究日趋成熟; 同时如今人工智能发展讯 速, 人工智能与时空数据挖掘也逐步融合。然而, 一方面, 目前时空大数据的处理分析和挖掘是一个 离散、迭代的过程, 通常需要针对不同的应用需求 场景提出不同的分析和挖掘算法并进行优化。因 此, 迫切需要一个集成众多时空分析和数据挖掘功 能的通用时空智能分析框架, 兼容多类模型和多源 时空数据, 从而实现时空大数据分析应用的统一化 和高效化。另一方面, 目前人工智能算法多为 “黑 箱模型”, 算法的不透明性导致难以对其进行扩展 和优化, 并行难度进一步加大。因此, 时空数据处 理分析算法的并行与底层人工智能算法的并行需 要进一步统一和融合。

\section{参考文献(References)}

程果, 景宁, 陈䒯, 等. 2012. 栅格数据处理中邻域型算法的 并行优化方法 [J]. 国防科技大学学报, 34(4): 114-119. [Cheng G, Jing N, Chen L, et al. 2012. Parallel optimization methods for raster data processing algorithms of neighborhood-scope[J]. Journal of National University of Defense Technology, 34(4): 114-119.]

杜江, 张铮, 张杰金金, 等. 2015. MapReduce 并行编程模型研 究综述 [J]. 计算机科学, 42(S1): 537-541. [Du J, Zhang Z, Zhang J X. 2015. Survey of MapReduce parallel programming model[J]. Computer Science, 42(S1): 537-541.]

贾婷, 魏祖宽, 唐曙光, 等. 2010. 一种面向并行空间查询的 数据划分方法 [J]. 计算机科学, 37(8): 198-200. [Jia T, Wei Z K, Tang S G, et al. 2010. New spatial data partition approach for spatial data query[J]. Computer Science, 37 (8): 198-200.]

隽志才, 倪安宁, 贾洪飞, 等. 2006. 两种策略下的最短路径 并行算法研究与实现 [J]. 系统工程理论方法应用, 15(2): 123-127. [Jun Z C, Ni A N, Jia H F, et al. 2006. Study and implement of shortest path parallel algorithms with two strategies $[\mathrm{J}]$. System Engineering- Theory Methodology Applications, 15(2): 123-127.]

雷德龙, 郭殿升, 陈崇成. 2014. 基于 MongoDB 的矢量空间 数据云存储与处理系统 $[\mathrm{J}]$. 地球信息科学学报, 16(4): 507-516. [Lei D L, Guo D S, Chen C C. 2014. Vector spatial data cloud storage and processing based on MongoDB [J]. Journal of Geo-information Science, 16(4): 507-516.] 李德仁, 马军, 邵振峰. 2015. 论时空大数据及其应用 $[\mathrm{J}]$. 卫 星应用, (9): 7-11. [Li D R, Ma J, Shao Z F. 2015. The application of spatial temporal big data[J]. Satellite Application, (9): 7-11.]

李德仁, 王树良, 李德毅. 2013. 空间数据挖掘理论与应用 [M]. 北京: 科学出版社. [Li D R, Wang S L, Li D Y. 2013. Spatial data mining theories and applications[M]. Beijing, China: Science Press.]

李德仁, 张良培, 夏桂松. 2014. 遥感大数据自动分析与数据 挖掘 [J]. 测绘学报, 43(12): 1211-1216. [Li D R, Zhang L P, Xia G S. 2014. Automatic analysis and mining of remote sensing big data[J]. Acta Geodaeticaet Cartographica Sinica, 43(12): 1211-1216.]

李建江, 崔健, 王聑, 等. 2011. MapReduce 并行编程模型研 究综述 [J]. 电子学报, 39(11): 2635-2642. [Li J J, Cui J, Wang D, et al. 2011. Survey of MapReduce parallel programming model[J]. Acta Electronica Sinica, 39(11): 2635-2642.] 李绍俊, 杨海军, 黄耀欢, 等. 2017. 基于 NoSQL数据库的空 间大数据分布式存储策略与实践 $[\mathrm{J}]$. 武汉大学学报: 信 息科学版, 42(2): 163-169. [Li S J, Yang H J, Huang Y H, et al. 2017. Geo-spatial big data storage based on NoSQL database[J]. Geomatics and Information Science of Wuhan University, 42(2): 163-169.]

廉捷. 2013. 基于用户特征的社交网络数据挖掘研究 [D]. 北 京: 北京交通大学. [Lian J. 2013. Research on user features based data mining in social networks[D]. Beijing, China: Beijing Jiaotong University.]

廖理. 2015. 基于 Neo4J 图数据库的时空数据存储 $[\mathrm{J}]$. 信息安 全与技术，6(8): 43-45. [Liao L. 2015. Application research of Neo4J in spatio-temporal data storage[J]. Information Security and Technology, 6(8): 43-45.]

刘润涛, 安晓华, 高晓爽. 2009. 一种基于 R-树的空间索引结 构 [J]. 计算机工程, 35(23): 32-34. [Liu R T, An X H, Gao X S. 2009. Spatial index structure based on R-tree[J]. Computer Engineering, 35(23): 32-34.]

卢风顺, 宋君强, 银福康, 等. 2011. CPU/GPU 协同并行计算 研究综述 [J]. 计算机科学, 38(3): 5-10. [Lu F S, Song J Q, Yin F K, et al. 2011. Survey of CPU/GPU synergetic parallel computing[J]. Computer Science, 38(3): 5-10.] 卢俊, 张保明, 黄薇, 等. 2009. 基于 GPU 的遥感像数据融合 
IHS 变换算法 [J]. 计算机工程, 35(7): 261-263. [Lu J, Zhang B M, Huang W, et al. 2009. IHS transform algorithm of remote sensing image data fusion based on GPU [J]. Computer Engineering, 35(7): 261-263.] 卢照, 师军. 2010. 并行最短路径搜索算法的设计与实现 [J]. 计算机工程与应用, 46(3): 69-71. [Lu Z, Shi J. 2010. Design and implementation of parallel shortest path search algorithm [J]. Computer Engineering and Applications, 46(3): 69-71.] 罗俊. 2016. 数据挖掘算法的并行化研究及其应用[D]. 青岛: 青岛大学, [Luo J. 2016. Research on parallelization of data mining algorithm and application[D]. Qingdao, China: Qingdao University.]

马林. 2009. 数据重现: 文件系统原理精解与数据恢复最佳 实践 $[M]$. 北京: 清华大学出版社. [Ma L. 2009. Shuju chongxian: Wenjian xitong yuanli jingjie yu shuju huifu zuijia shijian[M]. Beijing, China: Tsinghua University Press.]

马义松, 武志刚. 2016. 基于 $\mathrm{Neo} 4 \mathrm{~J}$ 的电力大数据建模及分析 [J]. 电工电能新技术, 35(2): 24-29. [Ma Y S, Wu Z G. 2016. Modeling and analysis of big data for power grid based on Neo4J[J]. Advanced Technology of Electrical Engineering and Energy, 35(2): 24-29.]

孟小峰, 慈祥. 2013. 大数据管理: 概念、技术与挑战 [J]. 计算 机研究与发展, 50(1): 146-169. [Meng X F, Ci X. 2013. Big data management: Concepts, techniques and challenges [J]. Journal of Computer Research and Development, 50 (1): 146-169.]

彭晓明, 郭浩然, 庞建民. 2012. 多核处理器: 技术、趋势和挑 战 [J]. 计算机科学, 39(Z3): 320-326. [Peng X M, Guo H R, Pang J M. 2012. Mutil-core processor: Technology, tendency and challenge[J]. Computer Science, 39(Z3): 320-326.]

田帅. 2013. 一种基于 MongoDB 和 HDFS 的大规模遥感数据 存储系统的设计与实现 [D]. 杭州: 浙江大学. [Tian S. 2013. A design and implementation of large-scale remote sensing data storage system based on MongoDB and HDFS [D]. Hangzhou, China: Zhejiang University.]

王鸿琰, 关雪峰, 吴华意. 2017. 一种面向 CPU/GPU 异构环 境的协同并行空间插值算法 [J]. 武汉大学学报: 信息科 学版, 42(12): 1688-1695. [Wang H Y, Guan X F, Wu H Y. 2017. A collaborative parallel spatial interpolation algorithm on oriented towards the heterogeneous CPU/GPU system[J]. Geomatics and Information Science of Wuhan University, 42(12): 1688-1695.]

王凯, 曹建成, 王乃生, 等. 2015. Hadoop 支持下的地理信息 大数据处理技术初探 $[\mathrm{J}]$. 测绘通报, (10): 114-117. [Wang K, Cao J C, Wang N S, et al. 2015. Research on GIS big data computing technologies based on Hadoop[J]. Bulletin of Surveying and Mapping, (10): 114-117.]

夏大文. 2016. 基于 MapReduce 的移动轨迹大数据挖掘方法
与应用研究 [D]. 重庆: 西南大学. [Xia D W. 2016. MapReduce: Based methodologies of mobile trajectory big data mining and its applications[D]. Chongqing, China: Southwest University.]

谢欢. 2015. 大数据挖掘中的并行算法研究及应用 [D]. 成都: 电子科技大学. [Xie H. 2015. Research and application on the parallel algorithm in big data mining[D]. Chengdu, China: University of Electronic Science and Technology of China.]

用密巧, 王占宏, 王志宇. 2017. 基于 Redis 的海量轨迹数据 存储模型研究 [J]. 微型电脑应用, 33(4): 9-11. [Yan M Q, Wang Z H, Wang Z Y. 2017. Large- scale trajectory data storage model based on Redis[J]. Microcomputer Applications, 33(4): 9-11.]

杨洪余, 李成明, 王小平, 等. 2017. CPU/GPU 异构环境下图 像协同并行处理模型 [J]. 集成技术, 6(5): 8-18. [Yang H Y, Li C M, Wang X P, et al. 2017. Image cooperative parallel processing model in CPU/GPU heterogeneous environment[J]. Journal of Integration Technology, 6(5): 8-18.]

杨靖宇, 张永生, 董广军. 2010. 基于 GPU 的遥感影像 SAM 分类算法并行化研究 [J]. 测绘科学, 35(3): 9-11. [Yang J Y, Zhang Y S, Dong G J. 2010. Investigation of parallel method of RS image SAM algorithmic based on GPU[J]. Science of Surveying and Mapping, 35(3): 9-11.]

殷进勇, 杨阳, 徐振朋, 等. 2015. 计算存储融合: 从高性能计 算到大数据 $[\mathrm{J}]$. 指挥控制与仿真, 37(3): 1-7. [Yin J Y, Yang Y, Xu Z P, et al. 2015. The fusion of computing and storage: From HPC to big data[J]. Command Control \& Simulation, 37(3): 1-7.]

尹芳, 冯敏, 诸云强, 等. 2013. 基于开源 Hadoop 的矢量空间 数据分布式处理研究 [J]. 计算机工程与应用, 49(16): 2529. [Yin F, Feng M, Zhu Y Q, et al. 2013. Research on vector spatial data distributed computing using Hadoop projects $[\mathrm{J}]$. Computer Engineering and Applications, 49(16): 25-29.]

张飞龙. 2016. 基于 MongoDB 遥感数据存储管理策略的研 究 [D]. 开封: 河南大学. [Zhang F L. 2016. Research on the storage management strategy of remote sensing data base on MongoDB[D]. Kaifeng, China: Henan University.] 张景云. 2013. 基于 Redis 的矢量数据组织研究 [D]. 南京: 南 京师范大学. [Zhang J Y. 2013. Vector data organization research based on Redis[D]. Nanjing, China: Nanjing Normal University.]

张晓兵. 2016. 基于 HBase 的弹性可视化遥感影像存储系统 [D]. 杭州: 浙江大学. [Zhang X B. 2016. An HBase based remote sensing elastic visualization storage system[D]. Hangzhou, China: Zhejiang University.]

赵永华, 迟学斌. 2005. 基于 SMP 集群的 MPI + OpenMP 混 
合编程模型及有效实现 $[\mathrm{J}]$. 微电子学与计算机, 22(10): 7-11. [Zhao Y H, Chi X B. 2005. MPI + OpenMP hybrid paradigms and efficient implementation base on SMP clusters[J]. Microelectronics \& Computer, 22(10): 7-11.]

郑坤, 付艳丽. 2015. 基于 HBase 和 GeoTools 的矢量空间数 据存储模型研究 [J]. 计算机应用与软件, 32(3): 23-26.

[Zheng K, Fu Y L. 2015. Research on vector spatial data storage model based on HBase and GeoTools[J]. Computer Applications and Software, 32(3): 23-26.]

朱效民, 潘景山, 孙占全, 等. 2013. 基于 OpenMP 的两个地 学基础空间分析算法的并行实现及优化 $[\mathrm{J}]$. 计算机科 学, 40(2): 8-11. [Zhu X M, Pan J S, Sun Z Q. 2013. Parallel implementation and optimization of two basic geospatial- analysis algorithms based on OpenMP[J]. Computer Science, 40(2): 8-11.]

Beaver D, Kumar S, Li H C, et al. 2010. Finding a needle in haystack: Facebook's photo storage[C]//Usenix conference on operating systems design and implementation. USENIX Association: 47-60.

Chang F, Dean J, Ghemawat S, et al. 2008. Bigtable: A distributed storage system for structured data[J]. ACM Transactions on Computer System. 26(2): 1-26.

Cheng B, Guan X F, Wu H Y, et al. 2016. Hypergraph+: An improved hypergraph- based task- scheduling algorithm for massive spatial data processing on master-slave platforms [J]. ISPRS International Journal of Geo-Information, 5(8): 141-157.

Chester S, Crowe J. Exploraions of parallel fp_growth[EB/OL]. 2011-08-13[2018-8-31]. http://webhome.csc.uvic.ca/schester/.

Dagum L, Menon R. 1998. OpenMP: An industry standard API for shared-memory programming[J]. IEEE Computational Science \& Engineering, 5(1): 46-55.

Dean J, Ghemawat S. 2004. MapReduce: Simplified data processing on large clusters[J]. Sixth Symposium on Operating System Design and Implementation, 51(1): 137-150.

Dinan J, Balaji P, Buntinas D, et al. 2016. An implementation and evaluation of the MPI 3.0 one-sided communication interface[J]. Concurrency and Computation: Practice and Experience, 28(17): 4385-4404.

Do H-T, Limet S, Melin E. 2011. Parallel computing flow accumulation in large digital elevation models[J]. Procedia Computer Science, 4(4): 2277-2286.

Garland M, Grand S L, Nickolls J, et al. 2008. Parallel computing experiences with CUDA[J]. IEEE Micro, 28(4): 13-27.

Ghemawat S, Gobioff H, Leung S T. 2003. The Google file system[J]. Proceedings of SOSP 2003, Operating Systems Review, 37(5): 29-43.

HDFS. 2012. HDFS architecture guide[EB/OL]. 2012-10-02
[2018- 08- 31]. http: //hadoop.apache.org/docs/hdfs/r0.22.0/ hdfs_design.html.

Hecht R, Jablonski S. 2012. NoSQL evaluation: A use case oriented survey[C]//International conference on cloud and service computing (ICSC). IEEE, 336-341.

Hong S, Oguntebi T, Olukotun K. 2011. Efficient parallel graph exploration on multi-core CPU and GPU[C]//International conference on parallel architectures and compilation techniques. IEEE Computer Society: 78-88.

Javier D, Camelia M-C, Alfonso N. 2012. A survey of parallel programming models and tools in the multi and many-core era[J]. IEEE Transactions on Parallel and Distributed System, 23(8): 1369-1386.

Langendoen H F. 1995. Parallelizing the polygon overlay problem using Orca[D]. Amsterdam, Holland: Vrije Universiteit Amsterdam.

Lanthier M, Nussbaum D, Sack J R. 2003. Parallel implementation of geometric shortest path algorithms[J]. Parallel Computing, 29(10): 1445-1479.

Li X, Li D R. 2014. Can night-time light images play a role in evaluating the Syrian Crisis[J]. International Journal of Remote Sensing, 35(18): 6648-6661.

Manyika J, Chui M, Brown B, et al. 2011. Big data: The next frontier for innovation, competition, and productivity[R]. Chicago, IL: The McKinsey Global Institute: 1-156.

Nickolls J, Dally W J. 2010. The GPU computing era[J]. IEEE Micro, 30(2): 56-69.

NoSQL. 2009. NoSQL definition: Next generation databases mostly addressing some of the points: Being non-relational, distributed, open-source and horizontally scalable[EB/ OL]. 2009-06-11[2018-08-31]. http://nosql-database.org/.

NVIDIA. 2017. NVIDIA Tesla V100 GPU architecture: The world's most advanced data center GPU[J/OL]. 2017-08-30 [2018- 08-31]. https://images.nvidia.com/content/volta- architecture/pdf/volta-architecture-whitepaper.pdf.

Qatawneh M, Sleit A, Almobaideen W. 2009. Parallel implementation of polygons clipping using transputer[J]. American Journal of Applied Sciences, 6(2): 214-218.

Qin C Z, Zhan L J. 2012. Parallelizing flow-accumulation calculations on graphics processing units: From iterative DEM preprocessing algorithm to recursive multiple-flowdirection algorithm[J]. Computers \& Geosciences, 43(6): 7-16.

Qin C Z, Zhan L J, Zhu A X, et al. 2014. A strategy for rasterbased geocomputation under different parallel computing platforms[J]. International Journal of Geographical Information Science, 28(11): 2127-2144.

Waldrop M. 2008. Big data: Wikiomics[J]. Nature, 455: 22-25. Wilson G V. 1994. Assessing the usability of parallel program- 
ming systems: The Cowichan problems[M]//Decker K M, Rehmann R M. Programming environments for massively parallel distributed systems. Basal, Switzerland: Birkhäuser: 183-193.

Wu H Y, Guan X F, Gong J Y. 2011. ParaStream: A parallel streaming delaunay triangulation algorithm for lidar points on multicore architectures[J]. Computers \& Geosciences, 37(9): 1355-1363.

Xu G H. 1999. Pay much attention to the digital earth by the social[J]. Science News Weekly, (1): 7-8.

Xu M, Cao H, Wang C Y. 2014. Raster-based parallel multiplicatively weighted voronoi diagrams algorithm with MapReduce [M]//Cao B Y, Ma S Q, Cao H H. Ecosystem assessment and fuzzy systems management. New York: Springer International Publishing: 177-188.

Zaharia M, Xin R S, Wendell P, et al. 2016. Apache spark: A unified engine for big data processing $[\mathrm{J}]$. Communications of the ACM, 59(11): 56-65.

Zhang T H, Zhu Z M, Gong W, et al. 2018. Estimation of ultrahigh resolution $\mathrm{PM}_{2.5}$ concentrations in urban areas using $160 \mathrm{~m}$ Gaofen-1 AOD retrievals[J]. Remote Sensing of Environment, 216(10): 91-104.

Zhao M, Cheng W M, Zhou C H, et al. 2018. Assessing spatiotemporal characteristics of urbanization dynamics in Southeast Asia using time series of DMSP/OLS nighttime light data[J]. Remote Sensing, 10(1): 47- 66.

\title{
Research progress and trends of parallel processing, analysis, and mining of big spatiotemporal data
}

\author{
GUAN Xuefeng, ZENG Yumei* \\ (State Key Laboratory of Information Engineering in Surveying, Mapping and Remote Sensing, \\ Wuhan University, Wuhan 430079, China)
}

\begin{abstract}
With the rapid development of the Internet, Internet of things, and cloud computing technology, data with geographical location and time tag are accumulated in an explosive way, and this indicates that we are in the era of big spatiotemporal data. In addition to the typical "4V" characteristics, big spatiotemporal data also contain rich semantic information and dynamic spatiotemporal patterns. Although massive spatiotemporal data have promoted the evolvement of various cross-disciplinary studies, traditional methods of data processing and analysis would no longer meet the requirements of efficient storage and real-time analysis of such data. Therefore, it is of great importance to integrate big spatiotemporal data with high-performance computing/cloud computing. To address this problem, this article begins with the concept and origin of big spatiotemporal data, and introduces its unique characteristics. Then, the performance requirements generated by current big data applications are analyzed, and the status quo of the underlying hardware and software is summarized. Furthermore, the article comprehensively reviews parallel processing, analysis, and mining methods for big spatiotemporal data. Finally, we conclude with the challenges and opportunities of storage, management, and parallel processing analysis of big spatiotemporal data.
\end{abstract}

Key words: big spatiotemporal data; high- performance computing; parallel spatial analysis; data mining; progress and trends 